\title{
A Family with Craniofrontonasal Syndrome and a Mutation (p.G151S) in the EFNB1 Gene: Expanding the Phenotype
}

\author{
Jaime Toral-López ${ }^{a}$ Luz M. González-Huerta ${ }^{b}$ Olga Messina Baas ${ }^{c}$ \\ Sergio A. Cuevas-Covarrubias ${ }^{b}$ \\ a Departamento de Genética Médica, Centro Médico Ecatepec, ISSEMYM, Ecatepec, and Departamentos de \\ ${ }^{b}$ Genética Médica and ' Oftalmología, Hospital General de México, Facultad de Medicina, Universidad Nacional \\ Autónoma de México (UNAM), Mexico City, Mexico
}

\section{Key Words}

Craniofrontonasal syndrome ·EFNB1 - Ephrin-B1 · p.G151S

mutation

\begin{abstract}
Craniofrontonasal syndrome (CFNS) is a rare genetic entity with X-linked dominant inheritance. CFNS is due to mutations in the Ephrin-B1 (EFNB1) gene. It is characterized by brachycephaly, frontonasal dysplasia, palate/lip defects, dental malocclusion, short neck, split nails, syndactyly, toe and finger defects, and minor skeletal defects. Intelligence is usually unaffected. CFNS exhibits unexpected manifestations between males and females as the latter are more affected. Cellular or metabolic interference due to X inactivation explains the more severe phenotype in heterozygous females. One family with several members affected with CFNS and 100 healthy controls were examined. DNA from leukocytes was isolated to analyze the EFNB1 gene. We did molecular modeling to assess the impact of the mutation on the EFNB1-encoded protein. DNA sequencing analysis of the EFNB1 gene of the affected members showed the heterozygous missense mutation c.451G $>\mathrm{A}$ in the EFNB1 gene (GRcH38, chrX:68,839,708; GERP score in hg38 of 9.961). This transition mutation resulted in the substitution of Gly at po-
\end{abstract}

sition 151 by Ser. Analysis of the healthy members of the family and 100 unrelated controls showed a normal sequence of the EFNB1 gene. Phenotypes of the patients in this family differ from the classical CFNS due to the decreased size of sulci and fissures, subarachnoid space and ventricles, and the absence of a cleft lip/palate.

(c) 2016 S. Karger AG, Basel

Craniofrontonasal syndrome (CFNS; OMIM 304110), a rare genetic syndrome with $\mathrm{X}$-linked dominant inheritance, is caused by loss-of-function mutations of EphrinB1 (EFNB1; OMIM 300035) [Wieland et al., 2004; Twigg et al., 2013]. CFNS consists of coronal synostosis and frontonasal dysplasia; moreover, palate/lip defects, dental malocclusion and a short neck are occasionally observed. Split nails are frequently present, whereas syndactyly, clinodactyly, toe and finger defects, and joint and minor skeletal defects are sometimes observed. Intelligence is usually normal [Saavedra et al., 1996; van den Elzen et al., 2014]. CFNS exhibits unexpected manifestations between males and females as the latter are more affected. Cellular or metabolic interference due to $\mathrm{X}$ inactivation is proposed to explain the more severe phenotype in heterozygous females. In the present study, we describe a Mexican

\section{KARGER}

E-Mail karger@karger.com

www.karger.com/msy
(C) 2016 S. Karger AG, Base

1661-8769/16/0071-0032\$39.50/0
Sergio Cuevas-Covarrubias

Departamento de Genética Médica

Hospital General de México, UNAM

Dr. Balmis 148 Col. Doctores, Mexico City 06726 (Mexico)

E-Mail sergiocuevasunam @ gmail.com 
family with CNFS and a mutation in the EFNB1 gene. In contrast to the features present in the 2 families previously reported, however, our family did not have cleft lip/ palate [Twigg et al., 2004; Özylmaz et al., 2015]. In addition, the family showed clinical data that were not previously observed in CFNS patients with mutations in the EFNB1 gene, representing an expanded phenotype.

\section{Materials and Methods}

\section{Patients}

The proband (case III-1), a 6-year-old girl, is the first child of healthy, nonconsanguineous young parents at the time of delivery. Family history was negative for intellectual disability or short stature. No history of prenatal exposure to teratogens or maternal illness was recorded. Following an uneventful pregnancy, the proband was born by caesarean section at 40 weeks' gestation with a weight of 2,800 $\mathrm{g}$ (10th percentile), height of $54 \mathrm{~cm}$ (97th percentile) and an OFC of $34 \mathrm{~cm}$ (10-25th percentile). Her Apgar scores were 7 and 8 at 1 and $5 \mathrm{~min}$, respectively. The neonate did not require special management. Her motor and language development has been normal. The parents reported that at the age of 5 years, 2 molars were easily fractured when chewing solid food. On physical examination in the present study, it was found that the patient weighed $17.6 \mathrm{~kg}$ (5th percentile), measured $112 \mathrm{~cm}$ in height (2550 th percentile) and had an OFC of $48 \mathrm{~cm}$ ( $<3 \mathrm{rd}$ percentile). She showed brachycephaly with temporary bulge, a broad metopic area with frontal bossing and lateral narrowing, facial asymmetry, ocular hypertelorism with a pupillary distance of $7.5 \mathrm{~cm}$, an intercanthal distance of $4.3 \mathrm{~cm}$, and upslanting palpebral fissures with a length of $3.1 \mathrm{~cm}$. She had a broad nasal bridge and a bifid nasal tip with a distance between nostrils of $3.5 \mathrm{~cm}$, a downslanting oral commissure (fig. 1B), a short neck, and split nails. Her toes exhibited wide spacing, distal hypoplasia and syndactyly of toes 2-3 (fig. 1C, D). Spinal column X-rays showed mild thoracic scoliosis, and a foot X-ray showed synostosis of the second to fourth interphalangeal joints of the left toes (fig. 1E). 3D CT showed plagiocephaly with a prominent front and flattened side, and absent left coronal suture. Brain MRI showed narrowing of the sulci and fissures, the subarachnoid space and the lateral and third ventricles (fig. 1F, G).

The mother (case II-3), a 26-year-old female, is the daughter of nonconsanguineous young parents, after a normal pregnancy, without consumption of teratogens or aggregated illnesses. Weight, size, and OFC at birth and further growth parameters were not recorded. She had had broken teeth since childhood when chewing solid food. Her intellectual development was normal. She weighed $44.5 \mathrm{~kg}$ ( $<3 \mathrm{rd}$ percentile), measured $141 \mathrm{~cm}$ in height ( $<3 \mathrm{rd}$ percentile) and had an OFC of $49 \mathrm{~cm}$ (<3rd percentile). She showed a brachycephalic skull with temporal bulging and frontal flattening, pinnae with dysplasia (antihelix-helix) and ocular hypertelorism with a pupillary distance of $8.5 \mathrm{~cm}$, intercanthal distance of $5.0 \mathrm{~cm}$ and palpebral fissures directed upwards with a length of $3.5 \mathrm{~cm}$. The eyeballs showed right divergent strabismus, and she had a broad nasal bridge and a bifid nasal tip (distance between nostrils: $3.5 \mathrm{~cm}$ ), a slightly downslanting oral commissure (fig. 1B), a short neck, and split nails. She had normal hands and feet.

Craniofrontonasal Syndrome: Expanding Phenotype
The brother of our proband (case III-2), a 4-year-old boy, showed skull dolichocephaly with slight hypertelorism and frontal bossing (fig. 1B). He weighed $13.8 \mathrm{~kg}(<3 \mathrm{rd}$ percentile), measured $100 \mathrm{~cm}$ in height (25-50th percentile) and had an OFC of $49 \mathrm{~cm}$ (3rd-10th percentile). Apparently, the maternal grandmother, maternal brother and a 7-month-old girl cousin had a similar phenotype as seen in our proband, but it was not possible to examine these patients.

\section{EFNB1 Gene Analysis}

After genomic DNA extraction using conventional methods, the EFNB1 gene was analyzed by PCR. The conditions and primers to amplify exons $1-5$ of the EFB1 gene were designed as follows: forward F1: 5'-AGAGCGACACCGAAGCCGGC-3'; reverse R1: 5'-CGCTCCTCCTCCGAAGACCT-3'; F2/3: 5'-GAGGCTGACCATCTTCTTCC-3'; R2/3: 5'-ACCCAGCCAGGAACATCTGT-3'; and F4/5: 5'-CCTGCCTCTCACCTGTTCTG-3'; R4/5: 5'-TACAAAGGTGGGCACAGCTG-3'. PCR products obtained from the proband, brother, mother, and 100 normal controls in the study were sequenced on an ABI 3730 automated sequencer (PE Biosystems, Foster City, Calif., USA).

\section{EFNB1 3D Protein Prediction}

Data on alignment of the protein were obtained from the RaptorX server [Källberg et al., 2012]; models were viewed in a SwissPdb Viewer, version 4.0, and homology modeling was carried out using SWISS-MODEL [Arnold et al., 2006].

\section{Results}

DNA analysis of the proband, her mother and her brother showed a normal amplification of exons 1-5. DNA sequencing analysis showed a c.451G $>$ A missense mutation within exon 3 of the EFNB1 gene (GRcH38, chrX: 68,839,708; GERP score in hg38 of 9.961; http:// genome.ucsc.edu/cgi-bin/hgTracks; fig. $1 \mathrm{H}$ ), which was previously reported as pathogenic. This mutation leads to a substitution of glycine at position 151 by a serine (p.G151S). Analysis of the unaffected members of the family and 100 healthy controls showed a normal sequence of the EFNB1 gene. No other nucleotide variations or polymorphisms were found in the rest of the analyzed exons.

\section{Modeling of EFNB1 (Ephrin-B1; Uniprot P98172)}

Protein

Structural change predictions of the mutant form of EFNB1 (p.G151S) revealed that the p.G151S mutation occurred between the $\beta-9$ chain and the $\alpha-3$ helix, which produced a change in the $\beta-12$ (it moved) and $\beta-14$ (lengthened by 3 amino acids) chains and formed a new $\beta-15$ chain in the final $C$-extreme. It was also observed that $\alpha-4$ was shortened by 1 amino acid, $\alpha-5$ moved and 


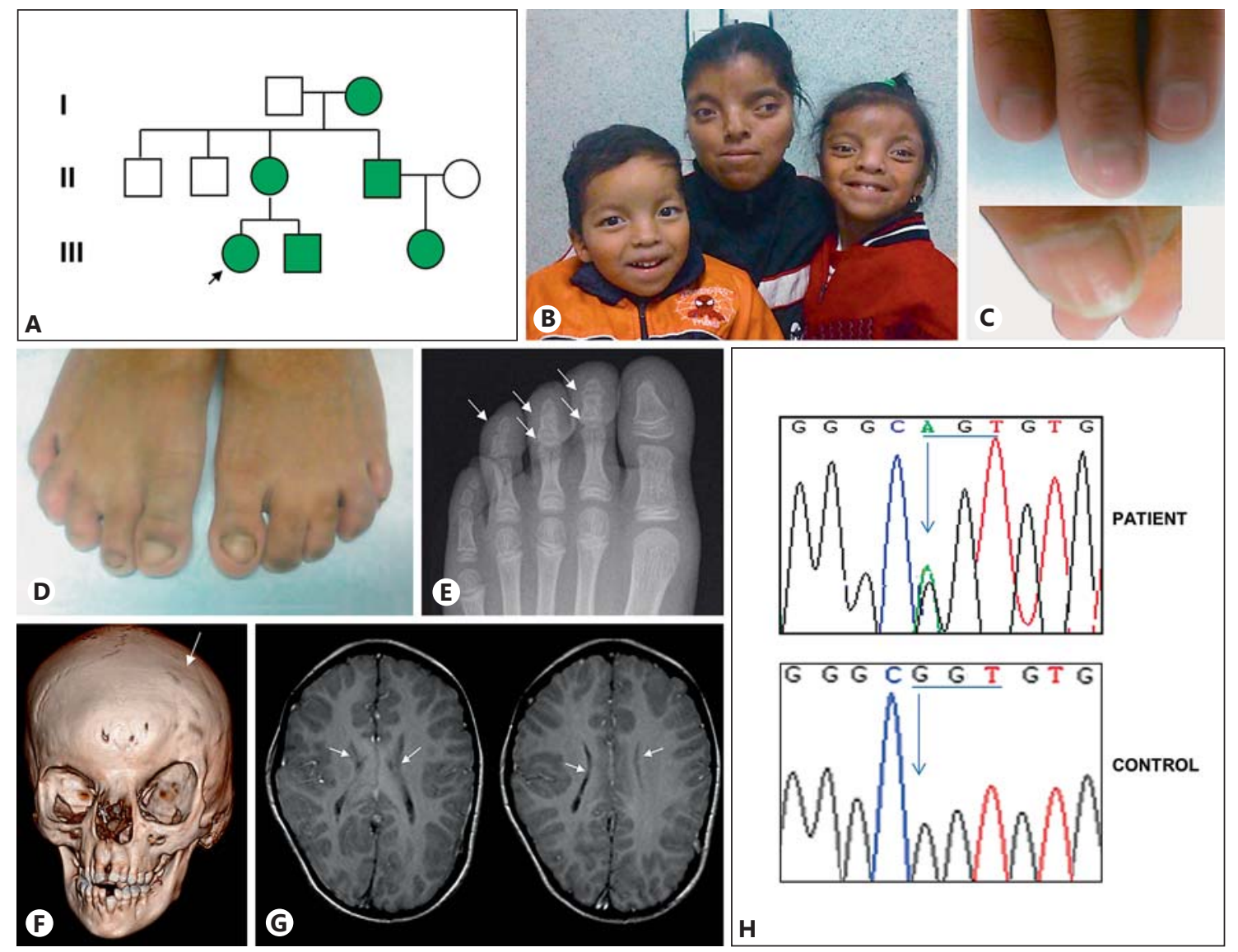

Fig. 1. A Pedigree of the family with mother, brother, grandmother, uncle, and cousin of our patient (arrow indicates index patient). B Facies of the family with CFNS. Note the slight facial asymmetry with frontal bossing, hypertelorism, and broad nasal bridge with bifid nasal tip. C Split nails in the proband and her mother. D Bilateral syndactyly of toes 2-3 in our proband. E Synostosis of the middle and distal interphalangeal joints of toes 2-4 (arrows). F Cranial 3D CT showing absence of the left coronal suture (ar- row). The ipsilateral orbit is elevated, retracted and medially rotated; there is frontal bossing and elevation of the craniosynostosis on the contralateral side. G Axial brain T2-weighted MRI showing parenchyma with grooves, lateral and third ventricle with significant narrowing (arrows). H Electropherogram of a partial sequence of the EFNB1 gene. The arrow shows the heterozygous change c. $451 \mathrm{G}>\mathrm{A}$ in the patient. a-6 helix was shortened by 2 amino acids, all of which caused a rotation and folding in the extracellular domain of the EFNB1 protein. Energy-computed analysis (the implementation of Swiss-Pdb Viewer on GROMOS96) of the alignment of EFNB1 amino acids with total electron density or electrostatic potential maps in the wild type represented $5,486.521 \mathrm{~kJ} / \mathrm{mol}$, whereas the mutant represented $4,598.287 \mathrm{~kJ} / \mathrm{mol}$. These data suggest that a mutation of p.G151S annuls the surface area of interaction in the extracellular domain with its Eph receptor due to a rotated and folded EFNB1 protein (fig. 2).

\section{Discussion}

Our CFNS family showed the p.G151S mutation previously found in 3 sporadic cases and 2 families [Young, 1987; Orr et al., 1997; Twigg et al., 2004; Özylmaz et al., 2015]. Clinically, our proband exhibited partial syndactyly between toes 2 and 3 of both feet, which was absent in the rest of the family, as described in the previous cases with the p.G151S mutation. Cleft lip/palate was absent in our family and in the 3 previous sporadic cases [Orr et al., 1997; Twigg et al., 2004]; nevertheless, it was present in 2 families with CFNS [Twigg et al., 2004; Özylmaz et al., 2015], denoting that the p.G151S mutation has clinical 

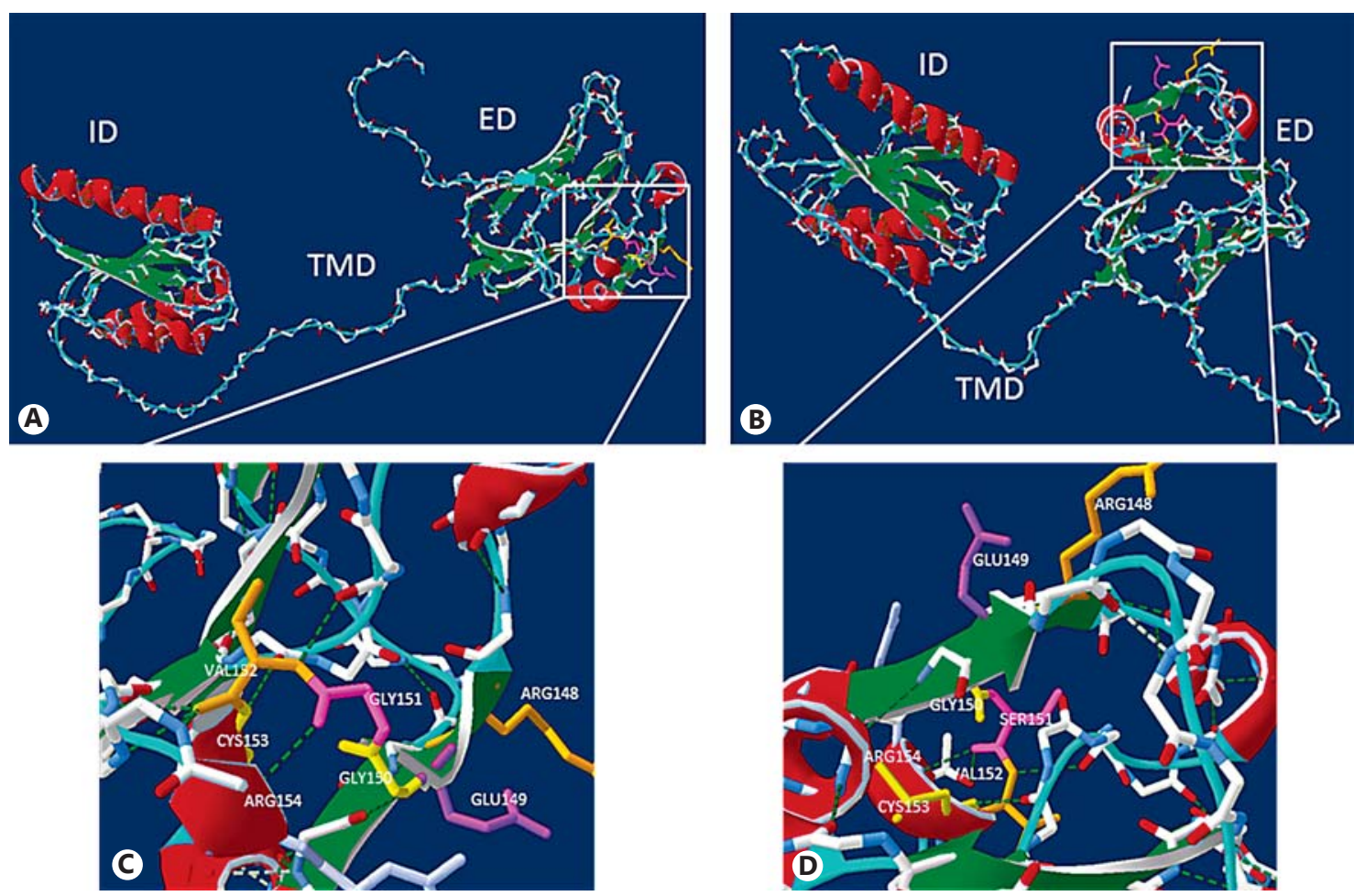

Fig. 2. A, B Wild-type and mutated EFNB1 protein. $\alpha$-Helices are in red, $\beta$-leaves in green, coil and loops in light blue, side chains of amino acids in white. B The mutated EFNB1 protein acquires a rotated and folded conformation of the extracellular domain. C, D Amplified (p.G151S) position change (pink) shows a strong gain of hydrogen bonds (dotted green lines). p.G151S neighboring amino acids are also shown (yellow and orange). ED = Extracellular domain; ID = intramembranous domain; TMD = transmembrane domain.

heterogeneity. Familial mutations in the EFNB1 gene have been observed in about $25 \%$, cleft lip/palate is present in $30 \%$ and cutaneous syndactyly of the hands and/or feet occurs in about $25 \%$ of the cases [Twigg et al., 2004, 2013]. Dental anomalies, such as crowding of the teeth, are observed in $23 \%$ of CFNS cases. In this study, we described brittle teeth in our proband and her mother, data that were not observed previously. A previous report suggested that EphB/Ephrin-B molecules are important for perivascular dental pulp stem cell migration toward the dentin surfaces and differentiation into functional odontoblasts; anomaly of Ephrin-B1 due to p.G151S mutation could be responsible for affecting the teeth [Arthur et al., 2009]. In addition, our proband showed synostosis of the interphalangeal joints of the left foot, and a decreased size of the sulci and fissures, subarachnoid space, lateral ventricles and third ventricle, data not previously reported in patients with CFNS and mutations in the EFNB1 gene. These clinical features could be considered in the phenotypic spectrum of patients with CFNS.

Craniofrontonasal Syndrome: Expanding Phenotype
The EFNB1 gene is located in Xq13.1, encodes the EFNB1 protein, comprises 5 exons and spans $13.17 \mathrm{~kb}$. The EFNB1 transmembrane ligand protein binds to Eph receptor tyrosine kinases, which are crucial for migration, adhesion and repulsion during neural, vascular and epithelial development. Loss of Ephrin-B1 leads to abnormal cartilage segmentation and the formation of additional skeletal elements [Compagni et al., 2003]. The X-linked dominant inheritance of CFNS is clinically unexpected because women are more severely affected than men; apparently, this stems from the random $\mathrm{X}$ inactivation in heterozygous females. Males with severe phenotypes similar to those of heterozygous women may have postzygous mutations of the EFNB1 gene brought about through the mechanism of metabolic or cell interference [Wieland et al., 2005; Twigg et al., 2013]. The inter- and intrafamilial clinical variability could correlate with the patchy or mosaic distribution of EFNB1 expression observed in the limbs of heterozygous mice with random $\mathrm{X}$ chromosome inactivation. Moreover, a marked EFNB1 overexpression 
in X chromosomes bearing the wild-type active allele has been reported [Compagni et al., 2003], suggesting that the malformation depends on the cells or tissues involved. Our modeling analysis showed a conformational change in the $\beta$-chains and $\alpha$-helices, which produced an abnormal folding and rotation of the extracellular domain of the EFNB1 protein; such data have not been shown in previous studies involving this p.G151S mutation.

Searching in the Data Browser of the Exome Variant Server (http://evs.gs.washington.edu/EVS/) and in the Human Gene Mutation Database (http://www.hgmd. cf.ac.uk/ac/index.php), we found that this particular mutation in the EFNB1 gene is present in CFNS showing clinical heterogeneity, including some cases with no clinical features [Özylmaz et al., 2015].

In contrast, the Grantham Matrix Score for the transition of glycine to serine is 56 , a relatively low score that suggests a limited effect of this mutation on the protein function [Grantham, 1974]. Nevertheless, the molecular modeling study indicated a highly significant impact; this analysis underscores the limitations of bioinformatic estimates of the effects of mutations.

In conclusion, this study described a family with CFNS and a p.G151S mutation in the EFNB1 gene with an ex- panded phenotype. In addition, the modeling protein showed that the p.G151S mutation produced an abnormal folding, which caused malfunction of the EFNB1 protein.

\section{Acknowledgments}

We would like to thank the family for participating in this study and the DGAPA-PAPIIT/Universidad Nacional Autónoma de México for the grant IN204114.

\section{Statement of Ethics}

The study protocol was approved by the Ethics Committee of the General Hospital of Mexico. Informed consent was obtained from the family.

\section{Disclosure Statement}

All authors declare no conflicts of interest.

\section{References}

Arnold K, Bordoli L, Kopp J, Schwede T: The SWISS-MODEL workspace: a web-based environment for protein structure homology modelling. Bioinformatics 22: 195-20 (2006).

- Arthur A, Koblar S, Shi S, Gronthos S: Eph/ ephrinB mediate dental pulp stem cell mobilization and function. J Dent Res 88:829-834 (2009).

Compagni A, Logan M, Klein R, Adams RH: Control of skeletal patterning by ephrinB1-EphB interactions. Dev Cell 5:217-230 (2003).

-Grantham R: Amino acid difference formula to help explain protein evolution. Science 185: 862-864 (1974).

Källberg M, Wang $\mathrm{H}$, Wang S, Peng j, Wang Z, et al: Template-based protein structure modeling using the RaptorX web server. Nat Protoc 7:1511-1522 (2012).
Orr DJA, Slaney S, Ashworth GJ, Poole MD: Craniofrontonasal dysplasia. Br J Plast Surg 50: 153-161 (1997).

Özylmaz B, Gezdirici A, Özen M, Kalenderer Ö Report of a family with craniofrontonasal syndrome. Clin Dysmorphol 2:79-83 (2015).

Saavedra D, Richieri-Costa A, Guion-Almeida ML, Cohen MM Jr: Craniofrontonasal syndrome: study of 41 patients. Am J Med Genet 61:147-151 (1996).

Twigg SR, Kan R, Babbs C, Bochukova EG, Robertson SP, et al: Mutations of ephrin-B1 (EFNB1), a marker of tissue boundary formation, cause craniofrontonasal syndrome. Proc Natl Acad Sci USA 101:8652-8657 (2004).

Twigg SR, Babbs C, van den Elzen ME, Goriely A, Taylor S, et al: Cellular interference in craniofrontonasal syndrome: males mosaic for mutations in the X-linked EFNB1 gene are more severely affected than true hemizygotes. Hum Mol Genet 22:1654-1662 (2013). van den Elzen ME, Twigg SR, Goos JA, Hoogeboom AJ, van den Ouweland AM, et al: Phenotypes of craniofrontonasal syndrome in patients with a pathogenic mutation in EFNB1. Eur J Hum Genet 22:995-1001 (2014).

Wieland I, Jakubiczka S, Muschke P, Cohen M, Thiele $\mathrm{H}$, et al: Mutations of the ephrin-B1 gene cause craniofrontonasal syndrome. Am J Hum Genet 74:1209-1215 (2004).

Wieland I, Reardon W, Jakubiczka S, Franco B, Kress W, et al: Twenty-six novel EFNB1 mutations in familial and sporadic craniofrontonasal syndrome (CFNS). Hum Mutat 26:113118 (2005).

Young ID: Craniofrontonasal dysplasia. J Med Genet 24:193-196 (1987). 